\title{
The anti-symmetric LS potential in flavor SU(3) limit from lattice QCD
}

\author{
Noriyoshi Ishii* \\ Kobe-branch, Center for Computational Sciences, University of Tsukuba \\ E-mail: ishiidribf.riken.70 \\ Keiko Murano \\ Yukawa Institute of Theoretical Physics, Kyoto University \\ E-mail: murano@yukawa.kyoto-u.ac.ip \\ Hidekatsu Nemura \\ Center for Computational Sciences, University of Tsukuba \\ E-mail: nemura.hidekatsu.gbeu.tsukuba.ac.jp

\section{Kenji Sasaki} \\ Center for Computational Sciences, University of Tsukuba \\ E-mail: kenjisehet.ph.tsukuba.ac.jp

\section{for HAL QCD Collaboration}

Hyperon-hyperon potentials in parity-odd sector are obtained together with the symmetric and the anti-symmetric spin-orbit potentials for irreducible representations $27 \oplus 10^{*} \oplus 10 \oplus 8$ in the flavor SU(3) limit. The results are converted to $\Lambda \mathrm{N}$ potentials to discuss the phenomenologically expected cancellation between the symmetric and the anti-symmetric LS potentials in $\Lambda \mathrm{N}$ sector.

31st International Symposium on Lattice Field Theory - LATTICE 2013

July 29 - August 3, 2013

Mainz, Germany

\footnotetext{
${ }^{*}$ Speaker.
} 


\section{Introduction}

The strong spin-orbit (LS) potential of NN interaction $V_{\mathrm{LS}}(r) \vec{L} \cdot\left(\vec{s}_{1}+\vec{s}_{2}\right)$ plays important roles in phenomenology such as the magic number of atomic nuclei and the p-wave neutron super fluidity in neutron stars [四]. In $\Lambda \mathrm{N}$ sector, since $\Lambda$ and $\mathrm{N}$ are not identical, the LS potential splits into two pieces, the $\Lambda$-spin dependent part and the nucleon-spin dependent part, i.e., $V_{\Lambda}(r) \vec{L} \cdot \vec{s}_{\Lambda}+V_{N}(r) \vec{L}$. $\vec{s}_{N}$. It is convenient to arrange these two terms as $V_{\mathrm{SLS}}(r) \vec{L} \cdot\left(\vec{s}_{\Lambda}+\vec{s}_{N}\right)+V_{\mathrm{ALS}}(r) \vec{L} \cdot\left(\vec{s}_{\Lambda}-\vec{s}_{N}\right)$, where the first and the second terms are referred to as the symmetric LS potential (SLS) and the antisymmetric LS potential (ALS), respectively. Whereas SLS conserves the total spin S, ALS changes the total spin, which provides a coupling between spin-singlet and spin-triplet sectors. For the NN sector, ALS does not exist, because the total spin $S$ is conserved due to the identical particle nature of nucleons. In contrast, ALS can exist for two-hyperon sector even in the flavor SU(3) limit, because a change of total spin $S$ can be compensated by a change of $8_{\mathrm{S}}$ and $8_{\mathrm{A}}$ of the irreducible representations (irreps.) of flavor $\mathrm{SU}(3)$.

High precision spectroscopy of hypernuclei reveals that spin-orbit splittings of $\Lambda$ in hypernuclei are small, which suggests the weakness of $\Lambda$-spin dependent part of the LS potential [0]. It can be restated that the strong ALS leads to the cancellation between ALS and SLS in $\Lambda \mathrm{N}$ interaction. There are several studies based on effective models. Quark model suggests a strong cancellation[园], whereas the meson exchange model suggests a weak cancellation[四].

The expected cancellation between SLS and ALS in $\Lambda \mathrm{N}$ sector may be affected by possible uncertainty from nuclear effects. It is therefore desirable to extract a direct information on SLS and ALS from $\Lambda \mathrm{N}$ scattering experiment, which however is difficult because of the short life time of hyperons. Lattice QCD (LQCD) first principle calculations may be used as an alternative. Recently,

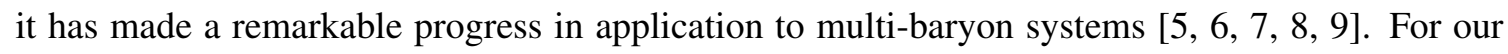
purpose, however, it is more appropriate to construct baryon-baryon potentials directly in LQCD. Recently HAL QCD collaboration has developed a method to construct potentials, which is faithful to the scattering phase shift, from Nambu-Bethe-Salpeter (NBS) wave functions [एU]]. The method has been first applied to NN potentials. and then employed not only to study other interactions such as $\mathrm{Y}$ (hyperon)N, $\mathrm{YY}, 3 \mathrm{~N}$, meson-meson and meson-baryons, but also to investigate properties of neutron star [5,6, 10-23]. Recently, the method is extended to calculate LS potentials and nuclear forces in the odd-parity sector [24]. We apply this method to the hyperon sector in the flavor SU(3) limit to consider the cancellation of ALS and SLS.

The contents are organized as follows. In Sect. \, we give a brief introduction to HAL QCD method of LQCD construction of baryon-baryon potentials. In Sect. B], we give details of LQCD set up of our numerical calculations. In Sect. 团, we give our numerical results.

\section{Formalism}

To define baryon-baryon potentials, we consider equal-time Nambu-Bethe-Salpeter (NBS) wave functions

$$
\psi_{\vec{k}}(\vec{x}-\vec{y}) \equiv Z_{B}^{-1}\langle 0|B(\vec{x}) B(\vec{y})| B(+\vec{k}) B(-\vec{k}), \text { in }\rangle,
$$

where $B(x)$ denotes an interpolating field for octet baryons, $Z_{B}$ denotes the normalization factor involved in the limit $B(\vec{x}, t) \rightarrow Z_{B}^{1 / 2} B_{\text {out }}(\vec{x}, t)$ as $t \rightarrow \infty$. It is possible to prove that equal-time NBS 
wave functions show the asymptotic long distance behavior as

$$
\psi_{\vec{k}}(\vec{r}) \sim e^{i \delta(k)} \frac{\sin (k|\vec{r}|+\delta(k))}{k|\vec{r}|}+\cdots, \quad \text { for } \quad|\vec{r}| \rightarrow \infty,
$$

where $\delta(k)$ denotes the scattering phase shift [ㅁ, $[25,[26]$. Note that this is exactly the same functional form as the scattering wave function in quantum mechanics. We define baryon-baryon potential $U\left(\vec{r}, \vec{r}^{\prime}\right)$ which is energy-independent and non-local so that the Schrödinger equation should reproduce equal-time NBS wave functions in the region $E_{\mathrm{CM}}<E_{\mathrm{th}} \equiv 2 m_{B}+m_{\mathrm{PS}}$ as

$$
\left(k^{2} / m_{B}-H_{0}\right) \psi_{\vec{k}}(\vec{r})=\int d^{3} r^{\prime} U\left(\vec{r}, \vec{r}^{\prime}\right) \psi_{\vec{k}}\left(\vec{r}^{\prime}\right) .
$$

The resultant potential $U\left(\vec{r}, \vec{r}^{\prime}\right)$ is faithful to the scattering phase because of Eq. (2.2]) [एU]]. (For explicit comparison between HAL QCD method and Lüscher's method, see Ref. [2]].)

The energy-independent potential $U\left(\vec{r}, \vec{r}^{\prime}\right)$ thus defined can efficiently be obtained by using "time-dependent" Schrödinger-like equation [ㅁ]]

$$
\left(\frac{1}{4 m_{B}} \frac{\partial^{2}}{\partial t^{2}}-\frac{\partial}{\partial t}-H_{0}\right) R(\vec{r}, t)=\int d^{3} r^{\prime} U\left(\vec{r}, \vec{r}^{\prime}\right) R\left(\vec{r}^{\prime}, t\right) .
$$

Here, $R(\vec{r}, t) \equiv C_{B B}(\vec{r}, t) / C_{B}(t)^{2}$ is a normalized four-point correlator of baryons, where $C_{B}(t)$ denotes a two-point correlator of baryon and $C_{B B}(\vec{r}, t) \equiv\langle 0|T[B(\vec{x}, t) B(\vec{y}, t) \bar{J}(t=0)]| 0\rangle$ denotes a four-point correlator of baryon associated with a two-baryon source operator $\bar{J}(t=0)$. Note that Eq. (2.4) allows us to obtain $U\left(\vec{r}, \vec{r}^{\prime}\right)$ even in the presence of of excited states [ए8]. In this paper, we use Eq. (2.4) after applying the derivative expansion $U\left(\vec{r}, \vec{r}^{\prime}\right)=V(\vec{r}, \vec{\nabla}) \delta^{3}\left(\vec{r}-\vec{r}^{\prime}\right)$ with

$$
\begin{aligned}
V(\vec{r}, \vec{\nabla}) \simeq & V_{\mathrm{C} ; S=0}(r) \mathbb{P}^{(S=0)}+V_{\mathrm{C} ; S=1}(r) \mathbb{P}^{(S=1)}+V_{\mathrm{T}}(r) S_{12} \\
& +V_{\mathrm{SLS}}(r) \vec{L} \cdot \vec{S}_{+}+V_{\mathrm{ALS}}(r) \vec{L} \cdot \vec{S}_{-}+O\left(\nabla^{2}\right),
\end{aligned}
$$

where $\mathbb{P}^{(S=0)} \equiv\left(1-\vec{\sigma}_{1} \cdot \vec{\sigma}_{2}\right) / 4$ and $\mathbb{P}^{(S=1)} \equiv\left(3+\vec{\sigma}_{1} \cdot \vec{\sigma}_{2}\right) / 4$ denote projection operators onto the spin-singlet and the spin-triplet sectors, respectively. $S_{12} \equiv 3\left(\hat{r} \cdot \vec{\sigma}_{1}\right)\left(\hat{r} \cdot \vec{\sigma}_{2}\right)-\vec{\sigma}_{1} \cdot \vec{\sigma}_{2}$ and $\vec{S}_{ \pm} \equiv$ $\left(\vec{\sigma}_{1} \pm \vec{\sigma}_{2}\right) / 2$.

\section{Numerical Setup}

Lattice QCD calculations are performed by using 3-flavor QCD gauge configuration generated by CP-PACS and JLQCD collaboration on $16^{3} \times 32$ lattice [27]. The gauge configurations are generated by employing renormalization group improved Iwasaki gauge action at $\beta=1.83$ and $O(a)$ improved Wilson quark (clover) action with $C_{\mathrm{SW}}=1.761$ at $\kappa_{\mathrm{uds}}=0.1371$ leading to $a=$ $0.121(2) \mathrm{fm}\left(a^{-1}=1630.58 \mathrm{MeV}\right), L=16 a \simeq 1.9 \mathrm{fm}, m_{\mathrm{PS}}=1013(1) \mathrm{MeV}$ and $m_{\mathrm{B}}=2051(3)$ $\mathrm{MeV}$. Baryon correlators are measured by imposing Dirichlet BC on $t=22$ for the source at $t=0$. We use eight source points to improve the statistic by temporally shifting gauge configurations. We also use charge-conjugation and time-reversal symmetry to double the number of data.

We measure the single baryon energy $E_{B}\left(\vec{p}^{2}\right)$ with non-vanishing spatial momentum $\vec{p}$, and find that the dispersion relation deviates from the relativistic form. We also find that $E_{B}\left(\vec{p}^{2}\right)$ in the low energy region $\vec{p}^{2} \leq 4(2 \pi / L)^{2}$ is well fitted with the functional form

$$
E_{B}^{2}\left(\vec{p}^{2}\right)=m_{B}^{2}+\alpha \vec{p}^{2}+O\left(\vec{p}^{4}\right),
$$


where $m_{B}=2040(4)$ and $\alpha=0.905(16)$ are obtained as fit parameters. For this functional form, the Eq. (2.4) is modified as

$$
\left(\frac{1}{\alpha}\left(\frac{1}{4 m_{B}} \frac{\partial^{2}}{\partial t^{2}}-\frac{\partial}{\partial t}\right)-H_{0}\right) R(\vec{r}, t)=\int d^{3} r^{\prime} U\left(\vec{r}, \vec{r}^{\prime}\right) R\left(\vec{r}^{\prime}, t\right) .
$$

In this paper, we present the potentials obtained at the time-slice $t=9$ from this modified equation.

To get four-point correlators of baryon, we employ the following four operators for the sink as

$$
\begin{aligned}
O_{\alpha \beta}^{(1)}(x, y) & \equiv \varepsilon_{c_{1} c_{2} c_{3}}\left[u_{c_{1}}^{T}(x) C \gamma_{5} d_{c_{2}}(x)\right] d_{c_{3} \alpha}(x) \cdot \varepsilon_{c_{4} c_{5} c_{6}}\left[s_{c_{4}}^{T}(y) C \gamma_{5} u_{c_{5}}(y)\right] d_{c_{6} \beta}(y) \\
O_{\alpha \beta}^{(2)}(x, y) & \equiv \varepsilon_{c_{1} c_{2} c_{3}}\left[u_{c_{1}}^{T}(x) C \gamma_{5} d_{c_{2}}(x)\right] u_{c_{3} \alpha}(x) \cdot \varepsilon_{c_{4} c_{5} c_{6}}\left[u_{c_{4}}^{T}(y) C \gamma_{5} d_{c_{5}}(y)\right] s_{c_{6} \beta}(y) \\
O_{\alpha \beta}^{(3)}(x, y) & \equiv \varepsilon_{c_{1} c_{2} c_{3}}\left[u_{c_{1}}^{T}(x) C \gamma_{5} d_{c_{2}}(x)\right] u_{c_{3} \alpha}(x) \cdot \varepsilon_{c_{4} c_{5} c_{6}}\left[d_{c_{4}}^{T}(y) C \gamma_{5} s_{c_{5}}(y)\right] u_{c_{6} \beta}(y) \\
O_{\alpha \beta}^{(4)}(x, y) & \equiv \varepsilon_{c_{1} c_{2} c_{3}}\left[u_{c_{1}}^{T}(x) C \gamma_{5} d_{c_{2}}(x)\right] u_{c_{3} \alpha}(x) \cdot \varepsilon_{c_{4} c_{5} c_{6}}\left[s_{c_{4}}^{T}(y) C \gamma_{5} u_{c_{5}}(y)\right] d_{c_{6} \beta}(y) .
\end{aligned}
$$

For the source, we employ the following four momentum wall source operators as

$$
\begin{aligned}
& \overline{\mathscr{J}}_{\alpha \beta}^{(1)}(\vec{p}) \equiv \sum_{x_{1}, \cdots, x_{6}} \varepsilon_{c_{1} c_{2} c_{3}}\left[\bar{u}_{c_{1}}\left(\vec{x}_{1}\right) C \gamma_{5} \bar{d}_{c_{2}}^{T}\left(\vec{x}_{2}\right)\right] \bar{d}_{c_{3} \alpha}\left(\vec{x}_{3}\right) \cdot \varepsilon_{c_{4} c_{5} c_{6}}\left[\bar{s}_{c_{4}}\left(\vec{x}_{4}\right) C \gamma_{5} \bar{u}_{c_{5}}^{T}\left(\vec{x}_{5}\right)\right] \bar{d}_{c_{6} \beta}\left(\vec{x}_{6}\right) \cdot e^{i \vec{p} \cdot\left(\vec{x}_{3}-\vec{x}_{6}\right)} \\
& \overline{\mathscr{J}}_{\alpha \beta}^{(2)}(\vec{p}) \equiv \sum_{x_{1}, \cdots, x_{6}} \varepsilon_{c_{1} c_{2} c_{3}}\left[\bar{u}_{c_{1}}\left(\vec{x}_{1}\right) C \gamma_{5} \bar{d}_{c_{2}}^{T}\left(\vec{x}_{2}\right)\right] \bar{u}_{c_{3} \alpha}\left(\vec{x}_{3}\right) \cdot \varepsilon_{c_{4} c_{5} c_{6}}\left[\bar{u}_{c_{4}}\left(\vec{x}_{4}\right) C \gamma_{5} \bar{d}_{c_{5}}^{T}\left(\vec{x}_{5}\right)\right] \bar{s}_{c_{6} \beta}\left(\vec{x}_{6}\right) \cdot e^{i \vec{p} \cdot\left(\vec{x}_{3}-\vec{x}_{6}\right)} \\
& \bar{J}_{\alpha \beta}^{(3)}(\vec{p}) \equiv \sum_{x_{1}, \cdots, x_{6}} \varepsilon_{c_{1} c_{2} c_{3}}\left[\bar{u}_{c_{1}}\left(\vec{x}_{1}\right) C \gamma_{5} \bar{d}_{c_{2}}^{T}\left(\vec{x}_{2}\right)\right] \bar{u}_{c_{3} \alpha}\left(\vec{x}_{3}\right) \cdot \varepsilon_{c_{4} c_{5} c_{6}}\left[\bar{d}_{c_{4}}\left(\vec{x}_{4}\right) C \gamma_{5} \bar{s}_{c_{5}}^{T}\left(\vec{x}_{5}\right)\right] \bar{u}_{c_{6} \beta}\left(\vec{x}_{6}\right) \cdot e^{i \vec{p} \cdot\left(\vec{x}_{3}-\vec{x}_{6}\right)} \\
& \overline{\mathscr{J}}_{\alpha \beta}^{(4)}(\vec{p}) \equiv \sum_{x_{1}, \cdots, x_{6}} \varepsilon_{c_{1} c_{2} c_{3}}\left[\bar{u}_{c_{1}}\left(\vec{x}_{1}\right) C \gamma_{5} \bar{d}_{c_{2}}^{T}\left(\vec{x}_{2}\right)\right] \bar{u}_{c_{3} \alpha}\left(\vec{x}_{3}\right) \cdot \varepsilon_{c_{4} c_{5} c_{6}}\left[\bar{s}_{c_{4}}\left(\vec{x}_{4}\right) C \gamma_{5} \bar{u}_{c_{5}}^{T}\left(\vec{x}_{5}\right)\right] \bar{d}_{c_{6} \beta}\left(\vec{x}_{6}\right) \cdot e^{i \vec{p} \cdot\left(\vec{x}_{3}-\vec{x}_{6}\right)},
\end{aligned}
$$

where the spatial momentum $\vec{p}$ is taken to be parallel and anti-parallel to the coordinate axes. These operators are used to construct $4 \times 4$ matrix correlator

$$
C_{\alpha \beta ; \alpha^{\prime} \beta^{\prime}}^{i, j}(\vec{x}-\vec{y}, t ; \vec{p}) \equiv\left\langle 0\left|T\left[O_{\alpha \beta}^{(i)}(\vec{x}, t, \vec{y}, t) \overline{\mathscr{J}}_{\alpha^{\prime} \beta^{\prime}}^{(j)}(\vec{p})\right]\right| 0\right\rangle .
$$

The matrix correlator is combined to give four-point correlators of octet-baryons in each of the flavor irreps. $27 \oplus 10^{*} \oplus 10 \oplus 8_{\mathrm{S}} \oplus 8_{\mathrm{A}}$. Analysis based on the cubic group shows that the "orbital component" of these source operators consists of $A_{1}^{+} \oplus E^{+} \oplus T_{1}^{-}$, which correspond roughly to $L=0, L=2, L=1$, respectively. These operators are used to generate four-point correlator of baryons for $J^{P}=0^{-}, 1^{-}$and $2^{-}$, which are used to obtain the potentials in parity-odd sector up to NLO by using Eq. (B.2). (See Ref. [24] for explicit procedure.)

\section{Numerical Results}

The irrep. 27 for parity-odd sector has only spin triplet component. The potential up to NLO is given by

$$
V^{(27)}(\vec{r}, \vec{\nabla}) \simeq V_{\mathrm{C} ; \mathrm{S}=1}^{(27)}(r) \mathbb{P}^{(S=1)}+V_{\mathrm{T}}^{(27)}(r) S_{12}+V_{\mathrm{SLS}}^{(27)}(r) \vec{L} \cdot \vec{S}_{+},
$$

with which Eq. (B.2) is solved for the sources $J^{P}\left({ }^{2 S+1} L_{J}\right)=0^{-}\left({ }^{3} P_{0}\right), 1^{-}\left({ }^{3} P_{1}\right), 2^{-}\left({ }^{3} P_{2}\right)$. Note that the irrep. 27 corresponds to the spin-triplet parity-odd sector of NN interaction. The result is shown in Fig. W(a). We see similar behaviors observed in Ref. [24] for the NN system such as (a.1) the 

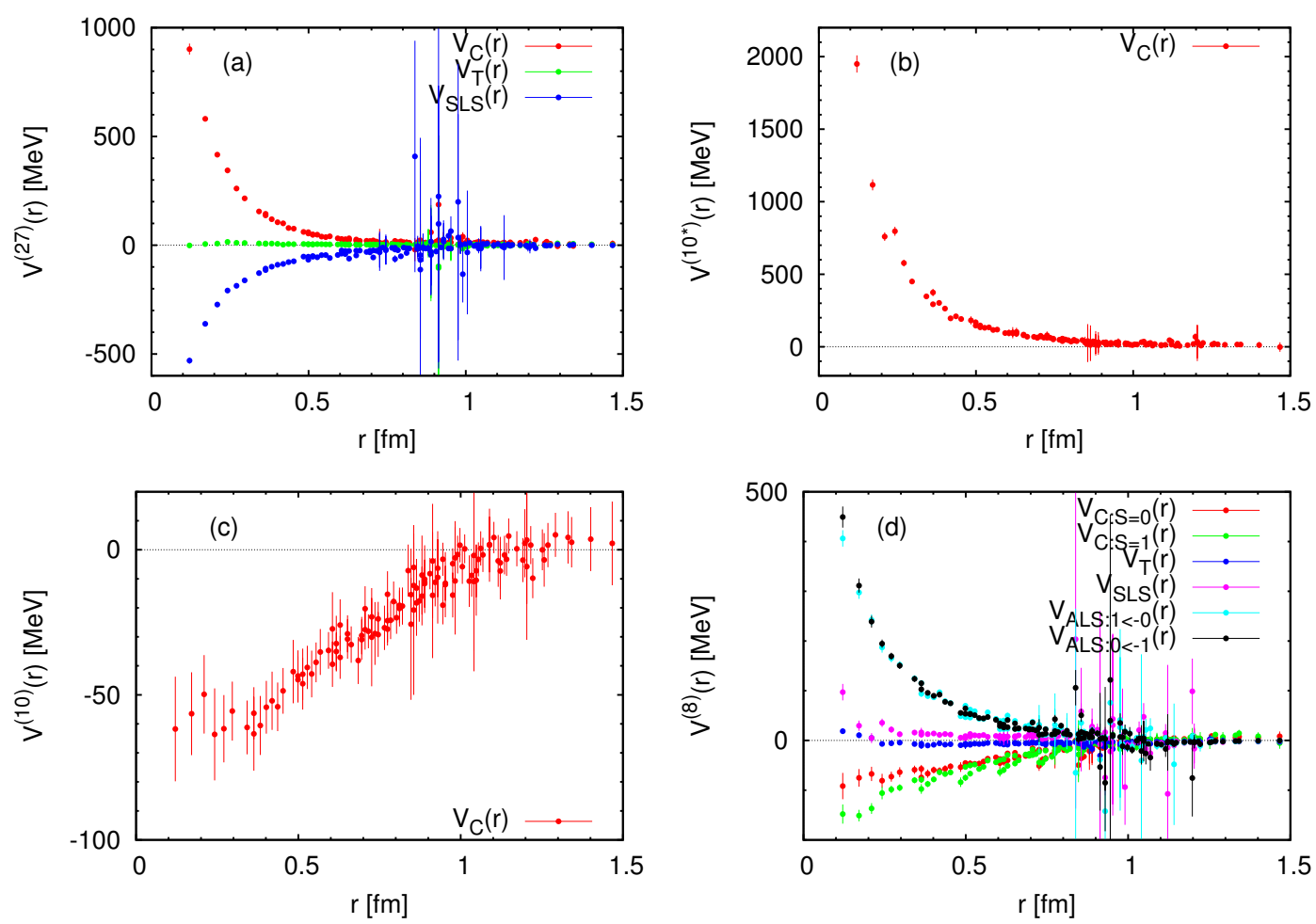

Figure 1: Parity-odd Potentials for irreps. (a) 27, (b) $10^{*}$, (c) 10 and (d) 8.

central potential has a repulsive core at short distance, (a.2) tensor potential is weak and positive, (a.3) LS potential is negative and strong.

The irrep. $10^{*}$ and 10 for parity-odd sector have only spin singlet component. The potential up to NLO is given by

$$
V^{(R)}(\vec{r}, \vec{\nabla}) \simeq V_{\mathrm{C} ; \mathrm{S}=0}^{(R)}(r) \mathbb{P}^{(S=0)} \quad \text { for } \quad R=10^{*}, 10,
$$

with which Eq. (3.2) is solved for the source $J^{P}\left({ }^{2 S+1} L_{J}\right)=1{ }^{-}\left({ }^{1} P_{1}\right)$. The results for irreps. $10^{*}$ and 10 are shown in Fig. $\mathbb{D}(\mathrm{b})$ and (c), respectively. Irrep. 10* corresponds to the spin-singlet parity-odd sector of $\mathrm{NN}$ interaction. We see that the central potential has a repulsive core at short distance. Irrep. 10 corresponds to parity-odd $\Sigma N(I=3 / 2)$ channel. We see that the central potential does not have a repulsive core, which is consistent with the quark model.

The irrep. 8 for parity-odd sector has both spin singlet and triplet components, where the coupling is provided by ALS. The potential up to NLO is given by

$$
\begin{aligned}
& V^{(8)}(\vec{r}, \vec{\nabla}) \\
& \simeq V_{\mathrm{C} ; \mathrm{S}=0}^{(8)}(r) \mathbb{P}^{(S=0)}+V_{\mathrm{C} ; \mathrm{S}=1}^{(8)}(r) \mathbb{P}^{(S=1)}+V_{\mathrm{T}}^{(8)}(r) S_{12}(\hat{r})+V_{\mathrm{SLS}}^{(8)}(r) \vec{L} \cdot \vec{S}_{+}+V_{\mathrm{ALS}}^{(8)}(r) \vec{L} \cdot \vec{S}_{-},
\end{aligned}
$$

with which Eq. (B.2) is solved for the source $J^{P}\left({ }^{2 S+1} L_{J}\right)=0^{-}\left({ }^{3} P_{0}\right), 1^{-}\left({ }^{3} P_{1}\right), 1^{-}\left({ }^{1} P_{1}\right), 2^{-}\left({ }^{3} P_{2}\right)$. The result is shown in Fig. W(d). (d.1) The central potentials do not have repulsive cores at short distance, which is consistent with quark model. (d.2) tensor potential and SLS are weak. (d.3) ALS is positive and strong, which is of comparable size as SLS for irrep. 27 sector. 
$\Lambda \mathrm{N}$ potential in parity-odd sector is obtained by making a linear combination of the potentials of irreps. $27,10^{*}$ and 8 in the following way:

$$
\begin{aligned}
V_{\Lambda N} & =\left(\frac{1}{2} V_{\mathrm{C} ; \mathrm{S}=0}^{\left(10^{*}\right)}(r)+\frac{1}{2} V_{\mathrm{C} ; \mathrm{S}=0}^{(8)}(r)\right) \mathbb{P}^{(S=0)}+\left(\frac{9}{10} V_{\mathrm{C} ; \mathrm{S}=1}^{(27)}(r)+\frac{1}{10} V_{\mathrm{C} ; \mathrm{S}=1}^{(8)}(r)\right) \mathbb{P}^{(S=1)} \\
& +\left(\frac{9}{10} V_{\mathrm{T}}^{(27)}(r)+\frac{1}{10} V_{\mathrm{T}}^{(8)}(r)\right) S_{12}+\left(\frac{9}{10} V_{\mathrm{SLS}}^{(27)}(r)+\frac{1}{10} V_{\mathrm{SLS}}^{(8)}(r)\right) \vec{L} \cdot \vec{S}_{+}+\frac{1}{2 \sqrt{5}} V_{\mathrm{ALS}}^{(8)}(r) \vec{L} \cdot \vec{S}_{-} .
\end{aligned}
$$

The result is shown in Fig. 1 . We see that SLS is negative and strong. This is natural because its main contribution comes from irrep. 27 with a factor of 9/10, i.e., it is almost the LS potential of NN interaction. On the other hand, ALS is positive and weak. Although ALS in irrep. 8 is originally as strong as SLS of irrep. 27, it is weakened by a Clebsch-Gordan factor $\frac{1}{2 \sqrt{5}}$. Although our data thus suggests weak cancellation of ALS and SLS, it is important to the light quark mass region taking into account the violation of the flavor SU(3) symmetry. It is also important to discuss the effect of possible $\Lambda-\Sigma$ mixing because our calculation is done in the flavor SU(3) limit.

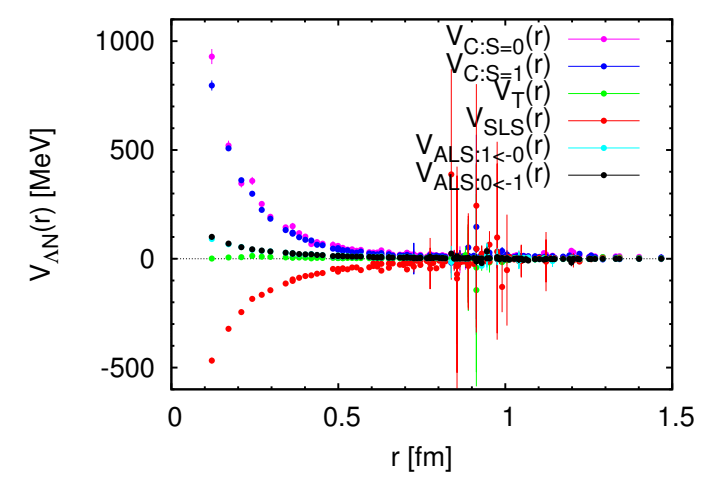

Figure 2: $\Lambda \mathrm{N}$ potential

\section{Summary}

We have presented the results of baryon-baryon potentials in parity-odd sector in the flavor SU(3) limit for irreducible representations (irreps.) of $27 \oplus 10^{*} \oplus 10 \oplus 8$. These results have been combined to give the $\Lambda \mathrm{N}$ potentials in parity-odd sector. We have seen that the symmetric LS potential (SLS) is negative and strong, whereas the anti-symmetric LS potential (ALS) is positive and weak. Thus we have obtained a weak cancellation between the symmetric and the anti-symmetric LS potentials. Because the calculation so far is performed in the heavy quark mass region in the flavor $\mathrm{SU}(3)$ limit, simulation with light $u, d$ quark masses, which introduces a violation of the flavor SU(3) symmetry will be required for a definite conclusion on the cancellation between SLS and ALS. In such simulations, contributions from $\Lambda-\Sigma$ mixing to the cancelation might be very different from the one in the flavor SU(3) limit.

We thank Prof. M. Oka and Prof. A. Umeya for fruitful discussions. Lattice QCD Monte Carlo calculation has been done on Blue Gene/Q at KEK. We thank CP-PACS and JLQCD Collaborations [D7] and ILDG/JLDG [D2] for dynamical QCD gauge configurations. We are grateful for authors 
and maintainers of CPS++ [28], a modified version of which is used in this calculation. This work is supported in part by Grant-in-Aid for Scientific Research (Nos. 24740144, 25105505, 25287046, 25400244) and SPIRE (Strategic Program for Innovative Research).

\section{References}

[1] P. Ring and P. Schuck, The Nuclear Many-Body Problem (Springer-Verlag, 1980) p. 1.

[2] H. Akikawa, S. Ajimura, R. E. Chrien, P. M. Eugenio, G. B. Franklin, J. Franz, L. Gang and K. Imai et al., Phys. Rev. Lett. 88 (2002) 082501.

[3] S. Takeuchi, O. Morimatsu, Y. Tani and M. Oka, Prog. Theor. Phys. Suppl. 137 (2000) 83.

[4] T. A. Rijken, V. G. J. Stoks and Y. Yamamoto, Phys. Rev. C 59 (1999) 21.

[5] N. Ishii, S. Aoki and T. Hatsuda, Phys. Rev. Lett. 99 (2007) 022001.

[6] T. Inoue et al. [HAL QCD Collaboration], Phys. Rev. Lett. 106 (2011) 162002.

[7] S. R. Beane et al. [NPLQCD Collaboration], Phys. Rev. Lett. 106 (2011) 162001.

[8] T. Yamazaki, K. -i. Ishikawa, Y. Kuramashi and A. Ukawa, Phys. Rev. D 86 (2012) 074514.

[9] S. R. Beane, E. Chang, S. D. Cohen, W. Detmold, H. W. Lin, T. C. Luu, K. Orginos and A. Parreno et al., Phys. Rev. D 87 (2013) 3, 034506.

[10] S. Aoki, T. Hatsuda and N. Ishii, Prog. Theor. Phys. 123 (2010) 89.

S. Aoki, T. Hatsuda and N. Ishii, Comput. Sci. Dis. 1 (2008) 015009.

[11] H. Nemura, N. Ishii, S. Aoki and T. Hatsuda, Phys. Lett. B 673 (2009) 136.

[12] T. Inoue et al. [HAL QCD Collaboration], Prog. Theor. Phys. 124 (2010) 591.

[13] T. Doi et al. [HAL QCD Collaboration], Prog. Theor. Phys. 127 (2012) 723.

[14] S. Aoki et al. [HAL QCD Collaboration], Proc. Japan Acad. B 87 (2011) 509.

[15] K. Murano, N. Ishii, S. Aoki and T. Hatsuda, Prog. Theor. Phys. 125 (2011) 1225.

[16] T. Inoue et al. [HAL QCD Collaboration], Nucl. Phys. A 881 (2012) 28.

[17] Y. Ikeda [HAL QCD Collaboration], PoS LATTICE 2011 (2011) 159.

[18] N. Ishii et al. [HAL QCD Collaboration], Phys. Lett. B 712 (2012) 437.

[19] S. Aoki, B. Charron, T. Doi, T. Hatsuda, T. Inoue and N. Ishii, Phys. Rev. D 87 (2013) 3, 034512.

[20] S. Aoki, N. Ishii, T. Doi, Y. Ikeda and T. Inoue, Phys. Rev. D 88 (2013) 014036.

[21] T. Kurth, N. Ishii, T. Doi, S. Aoki and T. Hatsuda, arXiv:1305.4462 [hep-lat].

[22] T. Inoue et al. [HAL QCD Collaboration], Phys. Rev. Lett. 111 (2013) 112503.

[23] S. Aoki et al. [HAL QCD Collaboration], PTEP 2012 (2012) 01 A105.

[24] K. Murano, N. Ishii, S. Aoki, T. Doi, T. Hatsuda, Y. Ikeda, T. Inoue and H. Nemura et al., arXiv:1305.2293 [hep-lat].

[25] C. J. D. Lin, G. Martinelli, C. T. Sachrajda and M. Testa, Nucl. Phys. B 619 (2001) 467.

[26] S. Aoki et al. [CP-PACS Collaboration], Phys. Rev. D 71 (2005) 094504.

[27] T. Ishikawa et al. [JLQCD Collaboration], Phys. Rev. D 78 (2008) 011502.

[28] Columbia Physics System (CPS), http://qcdoc.phys.columbia.edu/cps.html

[29] http://www.lqcd.org/ildg; http://www.jldg.org/ 